\title{
Topological Structure of Non-separable Sigma-locally Compact Convex Sets
}

by

\section{Iryna BANAKH, Taras BANAKH and Katsuhisa KOSHINO \\ Presented by Czesław BESSAGA}

Summary. For an infinite cardinal $\kappa$ let $\ell_{2}^{f}(\kappa)$ be the linear hull of the standard othonormal base of the Hilbert space $\ell_{2}(\kappa)$ of density $\kappa$. We prove that a non-separable convex subset $X$ of density $\kappa=\operatorname{dens}(X)$ in a locally convex linear metric space is homeomorphic to the space

- $\ell_{2}^{f}(\kappa)$ if and only if $X$ can be written as a countable union of finite-dimensional locally compact subspaces;

- $\mathbb{I}^{\omega} \times \ell_{2}^{f}(\kappa)$ if and only if $X$ contains a topological copy of the Hilbert cube $\mathbb{I}^{\omega}$ and $X$ can be written as a countable union of locally compact subspaces.

For an infinite cardinal $\kappa$ let

$$
\ell_{2}(\kappa)=\left\{\left(x_{i}\right)_{i \in \kappa} \in \mathbb{R}^{\kappa}: \sum_{i \in \kappa}\left|x_{i}\right|^{2}<\infty\right\}
$$

be the standard Hilbert space of density $\kappa$, endowed with the norm $\left\|\left(x_{i}\right)_{i \in \kappa}\right\|$ $=\left(\sum_{i \in \kappa}\left|x_{i}\right|^{2}\right)^{1 / 2}$, and

$$
\ell_{2}^{f}(\kappa)=\left\{\left(x_{i}\right)_{i \in \kappa} \in \ell_{2}(\kappa):\left|\left\{i \in \kappa: x_{i} \neq 0\right\}\right|<\infty\right\}
$$

be the linear hull of the standard orthonormal basis in $\ell_{2}(\kappa)$. For the smallest infinite cardinal $\kappa=\omega$ the spaces $\ell_{2}(\omega)$ and $\ell_{2}^{f}(\omega)$ are denoted by $\ell_{2}$ and $\ell_{2}^{f}$, respectively. We shall by $\mathbb{I}$ denote the closed unit interval $[0,1]$ and by $\mathbb{I}^{\omega}$ the Hilbert cube. A closed subset $A$ of a topological space $X$ is called a $Z$-set in $X$ if the set $\left\{f \in C\left(\mathbb{I}^{\omega}, X\right): f\left(\mathbb{I}^{\omega}\right) \cap A=\emptyset\right\}$ is dense in the function space $C\left(\mathbb{I}^{\omega}, X\right)$ endowed with the compact-open topology. Let $A \subset B$ be

2010 Mathematics Subject Classification: 57N17, 52A07.

Key words and phrases: convex set, pre-Hilbert space, homeomorphic. 
two subsets in a linear space $L$. We shall say that $A$ has infinite codimension in $B$ if the linear hull $\operatorname{lin}(A)$ has infinite codimension in $\operatorname{lin}(B)$.

The following characterization of convex sets homeomorphic to $\ell_{2}^{f}$ or $\mathbb{I}^{\omega} \times \ell_{2}^{f}$ has been obtained due to combined efforts of T. Dobrowolski [6], D. Curtis, T. Dobrowolski and J. Mogilski [5], and T. Banakh [1] (see also Theorem 5.3.12 and 5.3.2 in [3]).

THEOREM 1. A convex subset $X$ of a locally convex linear metric space is homeomorphic to the space

(1) $l_{2}^{f}$ if and only if $X$ is infinite-dimensional and $X$ can be written as a countable union of finite-dimensional compact sets;

(2) $\mathbb{I}^{\omega} \times l_{2}^{f}$ if $X$ can be written as a countable union of compact $Z$-sets and $X$ contains a subset $Q$ which is homeomorphic to the Hilbert cube and has infinite codimension in $X$.

In this paper we shall prove a non-separable counterpart of Theorem 1 . For a topological space $X$ its density dens $(X)$ is the smallest cardinality $|D|$ of a dense subset $D \subset X$.

Theorem 2. A non-separable convex subset $X$ of density $\kappa=\operatorname{dens}(X)$ in a locally convex linear metric space is homeomorphic to the space

(1) $\ell_{2}^{f}(\kappa)$ if and only if $X$ can be written as a countable union of finitedimensional locally compact spaces;

(2) $\mathbb{I}^{\omega} \times \ell_{2}^{f}(\kappa)$ if and only if $X$ contains a subspace homeomorphic to the Hilbert cube $\mathbb{I}^{\omega}$ and $X$ can be written as a countable union of locally compact spaces.

In fact, Theorem 2 follows from a more general Theorem 3 below characterizing pairs of convex sets homeomorphic to the pairs $\left(\ell_{2}(\kappa), \ell_{2}^{f}(\kappa)\right)$ or $\left(\mathbb{I}^{\omega} \times \ell_{2}(\kappa), \mathbb{I}^{\omega} \times \ell_{2}^{f}(\kappa)\right)$. We say that for topological spaces $A \subset X$ and $B \subset Y$ the pairs $(X, A)$ and $(Y, B)$ are homeomorphic if there is a homeomorphism $h: X \rightarrow Y$ such that $h(A)=B$. By a Fréchet space we understand a locally convex linear complete metric space.

THEOREM 3. Let $X$ be a non-separable convex set of density $\kappa=\operatorname{dens}(X)$ in a Fréchet space $L$, and $\bar{X}$ be the closure of $X$ in $L$. The pair $(\bar{X}, X)$ is homeomorphic to the pair

(1) $\left(\ell_{2}(\kappa), \ell_{2}^{f}(\kappa)\right)$ if and only if $X$ can be written as a countable union of finite-dimensional locally compact spaces;

(2) $\left(\mathbb{I}^{\omega} \times \ell_{2}(\kappa), \mathbb{I}^{\omega} \times \ell_{2}^{f}(\kappa)\right)$ if and only if $X$ contains a topological copy of the Hilbert cube $\mathbb{I}^{\omega}$ and $X$ can be written as a countable union of locally compact spaces. 
Theorem 3 will be derived from the following two results, first of which is due to T. Banakh and R. Cauty [2].

TheOrem 4 (Banakh, Cauty). Each non-separable closed convex set $X$ in a Fréchet space is homeomorphic to the Hilbert space $\ell_{2}(\kappa)$ of density $\kappa=\operatorname{dens}(X)$.

The other result used in the proof of Theorem 3 is a topological characterization of the pairs $\left(\ell_{2}(\kappa), \ell_{2}^{f}(\kappa)\right)$ and $\left(\mathbb{I}^{\omega} \times \ell_{2}(\kappa), \mathbb{I}^{\omega} \times \ell_{2}^{f}(\kappa)\right)$ due to J. West [10] (see also [9] and [7]).

Theorem 5 (West). A pair $(X, Y)$ of topological spaces $Y \subset X$ is homeomorphic to $\left(\mathbb{I}^{\omega} \times \ell_{2}(\kappa), \mathbb{I}^{\omega} \times \ell_{2}^{f}(\kappa)\right)$ (resp. $\left.\left(\ell_{2}(\kappa), \ell_{2}^{f}(\kappa)\right)\right)$ for an infinite cardinal $\kappa$ if and only if

(1) $X$ is homeomorphic to $\ell_{2}(\kappa)$;

(2) $Y$ can be written as a countable union of (finite-dimensional) locally compact spaces; and

(3) $Y$ absorbs (finite-dimensional) compact subsets of $X$ in the sense that for each compact (finite-dimensional) subset $K \subset X$, a compact subset $B \subset K \cap Y$, and an open cover $\mathcal{U}$ of $X$ there is a topological embedding $h: K \rightarrow Y$ such that $h|B=\mathrm{id}| B$ and $h$ is $\mathcal{U}$-near to the identity embedding id : $K \rightarrow X$.

Given a cover $\mathcal{U}$ of a topological space $X$, we say that two maps $f, g$ : $Z \rightarrow X$ are $\mathcal{U}$-near and denote this by $(f, g) \prec \mathcal{U}$ if for each point $z \in Z$ the doubleton $\{f(z), g(z)\}$ is contained in some set $U \in \mathcal{U}$.

Proof of Theorem 3 . The "only if" parts in both statements of Theorem 3 are trivial. To prove the "if" part, assume that $X$ is a non-separable convex subset of a Fréchet space $L$ and let $\bar{X}$ be the closure of $X$ in $L$. By Theorem 4 , the space $\bar{X}$ is homeomorphic to the Hilbert space $\ell_{2}(\kappa)$ of density $\kappa=$ $\operatorname{dens}(\bar{X})=\operatorname{dens}(X)$. Now we consider two cases.

1) Assume that the convex set $X$ can be written as a countable union of finite-dimensional locally compact spaces. By Theorem 5, the existence of a homeomorphism of $(\bar{X}, X)$ and $\left(\ell_{2}(\kappa), \ell_{2}^{f}(\kappa)\right)$ will follow as soon as we check that $X$ absorbs finite-dimensional compact subsets of $\bar{X}$. Fix a finitedimensional compact subset $K \subset \bar{X}$, a compact subset $B \subset K \cap X$, and an open cover $\mathcal{U}$ of $\bar{X}$. By the density of $X$ in $\bar{X}$ and the separability of $K$, there is a separable convex subset $Y \subset X$ such that $B \subset Y$ and $K \subset \bar{Y}$. Moreover, using the fact that $X$ is not separable, we can choose $Y$ so that its closure $\bar{Y}$ is not locally compact. By Theorem 4.4 of [5], the pair $(\bar{Y}, \bar{Y} \cap X)$ is homeomorphic to $\left(\ell_{2}, \ell_{2}^{f}\right)$, and by Theorem $5, \bar{Y} \cap X$ absorbs finite-dimensional compact subsets of $\bar{Y}$. Consequently, for the finite-dimensional compact subset $K \subset \bar{Y} \subset \bar{X}$ there is a topological embedding $f: K \rightarrow \bar{Y} \cap X \subset X$ such 
that $f|B=\mathrm{id}| B$ and $f$ is $\mathcal{U}$-near to the identity embedding $K \rightarrow \bar{Y} \subset \bar{X}$. This means that $X$ absorbs finite-dimensional compact subsets of $\bar{X}$. By Theorem 5. $(\bar{X}, X)$ is homeomorphic to $\left(\ell_{2}(\kappa), \ell_{2}^{f}(\kappa)\right)$.

2) Next, assume that $X$ contains a subspace $A \subset X$ homeomorphic to the Hilbert cube and $X$ can be written as a countable union of locally compact subsets. By Theorem 5, the existence of a homeomorphism of $(\bar{X}, X)$ and $\left(\mathbb{I}^{\omega} \times \ell_{2}(\kappa), \mathbb{I}^{\omega} \times \ell_{2}^{f}(\kappa)\right)$ will follow as soon as we check that $X$ absorbs compact subsets of $\bar{X}$. Fix a compact subset $K \subset \bar{X}$, a compact subset $B \subset K \cap X$, and an open cover $\mathcal{U}$ of $\bar{X}$. Using the density of $X$ in $\bar{X}$ and the separability of the compact set $K \cup A$, we can find a separable convex subset $Y \subset X$ of $X$ such that $A \cup B \subset Y$ and $K \subset \bar{Y}$. We can assume that $Y=\bar{Y} \cap X$. Since $X$ is not separable, the compact set $A$ has infinite codimension in $X$. So we can choose $Y$ to be so large that $A$ has infinite codimension in $Y$ and $\bar{Y}$ is not locally compact. By Proposition 3.1 of [5], each closed locally compact subset of $\bar{Y}$ is a $Z$-set in $\bar{Y}$. It follows that the separable convex set $Y=\bar{Y} \cap X$ is a countable union of compact $Z$-sets. Since the topological copy $A$ of the Hilbert cube has infinite codimension in $Y$, the convex set $Y$ is homeomorphic to $\mathbb{I}^{\omega} \times \ell_{2}^{f}$ by Theorem $1(2)$. By the Uniqueness Theorem for Skeletoids [4, Theorem 2.1], the pair $(\bar{Y}, Y)$ is homeomorphic to $\left(\mathbb{I}^{\omega} \times \ell_{2}, \mathbb{I}^{\omega} \times \ell_{2}^{f}\right)$, and by Theorem 5, $Y$ absorbs compact subsets of $\bar{Y}$. In particular, for the compact subset $K \subset \bar{Y}$ there is a topological embedding $f: K \rightarrow Y \subset X$ such that $f|B=\mathrm{id}| B$ and $(f, \mathrm{id}) \prec \mathcal{U}$. This means that $X$ absorbs compact subsets of $\bar{X}$, and $(\bar{X}, X)$ is homeomorphic to $\left(\mathbb{I}^{\omega} \times \ell_{2}(\kappa), \mathbb{I}^{\omega} \times \ell_{2}^{f}(\kappa)\right)$ according to Theorem 5. This completes the proof of Theorem 3 .

Remark 1. By Proposition 3.2 of [8], each infinite-dimensional convex subset $X$ of a Fréchet space absorbs finite-dimensional compact subsets of its closure $\bar{X}$. This fact implies Theorems 2(1) and 3(1) (see [8]). It should be mentioned that [8] also contains a result a bit weaker than Theorem $3(2)$. In fact, the present paper is a unification of two papers, one prepared by the first two authors and the other by the third author. The results of these papers were obtained independently and almost at the same time.

We do not know if the condition on $Q$ to have infinite codimension in $X$ in Theorem 1(2) can be omitted.

Problem 1. Assume that a subset $A$ of a Fréchet space is homeomorphic to the Hilbert cube $\mathbb{I}^{\omega}$. Does $A$ contain a subset $B$ homeomorphic to the Hilbert cube and of infinite codimension in $A$ ?

\section{References}

[1] T. Banakh, The strongly universal property in closed convex sets, Mat. Stud. 10 (1998), 203-218. 
[2] T. Banakh and R. Cauty, Topological classification of convex sets in Fréchet spaces, Studia Math. 205 (2011), 1-11.

[3] T. Banakh, T. Radul and M. Zarichnyi, Absorbing Sets in Infinite-Dimensional Manifolds, VNTL Publ., Lviv, 1996, 240 pp.

[4] C. Bessaga and A. Pełczyński, Selected Topics in Infinite-Dimensional Topology, PWN, Warszawa, 1975.

[5] D. Curtis, T. Dobrowolski and J. Mogilski, Some applications of the topological characterizations of the sigma-compact spaces $l_{f}^{2}$ and $\Sigma$, Trans. Amer. Math. Soc. 284 (1984), 837-846.

[6] T. Dobrowolski, The compact Z-set property in convex sets, Topology Appl. 23 (1986), 163-172.

[7] K. Koshino, Characterizing non-separable sigma-locally compact infinite-dimensional manifolds and its applications, J. Math. Soc. Japan, to appear.

[8] K. Koshino, The topological types of non-separable sigma-locally compact convex sets, preprint, 2013; included in PhD dissertation, Univ. of Tsukuba, 2014.

[9] K. Sakai and M. Yaguchi, Characterizing manifolds modeled on certain dense subspaces of non-separable Hilbert spaces, Tsukuba J. Math. 27 (2003), 143-159.

[10] J. West, The ambient homeomorphy of an incomplete subspace of infinite-dimensional Hilbert spaces, Pacific J. Math. 34 (1970) 257-267.

Iryna Banakh

Institute for Applied Problems of

Mechanics and Mathematics

of Ukrainian Academy of Sciences

Naukova 3b

Lviv, Ukraine

E-mail: ibanakh@yahoo.com

Katsuhisa Koshino

Doctoral Program in Mathematics

Graduate School of Pure and Applied Sciences

University of Tsukuba

Tsukuba, 305-8571, Japan

E-mail: kakoshino@math.tsukuba.ac.jp
Taras Banakh Ivan Franko National University of Lviv Lviv, Ukraine and Jan Kochanowski University Kielce, Poland E-mail: t.o.banakh@gmail.com

Received March 13, 2013;

received in final form September 24, 2013 
